\title{
Calibration transfer between short wave near infrared photodiode array instruments
}

\author{
C. Hayes,${ }^{\mathrm{a} *} \mathrm{~K}$. Walsh ${ }^{\mathrm{b}}$ and R. Lerud ${ }^{\mathrm{c}}$ \\ ${ }^{a}$ Central Queensland University, Rockhampton, QLD, 4702, Australia. E-mail: c.hayes@cqu.edu.au \\ ${ }^{b}$ Central Queensland University, Rockhampton, QLD, 4702, Australia. E-mail: $\overline{k . w a l s h @ c q u . e d u . a u ~}$ \\ cPortland State University, Portland, OR, 97203, USA. E-mail: lerud@pdx.edu
}

Transfer methods were compared for the porting of partial least squares models for intact mango dry matter content between short wave near infrared silicon photodiode array instruments. Methods included bias adjustment using average difference spectrum, new pixel-to-wavelength assignments, piecewise direct standardisation (PDS), global models, model updating (MU) and combinations of these. Best results ( $R^{2}>0.84$ and bias $<0.2$ ) were obtained by PDS using the same variety of fruit in calibration and transfer sets. The use of an apple spectra transfer set was also successful, if the wavelength accuracy of the slave unit(s) is satisfactory. Alternatively, a field practical solution that gave acceptable prediction results involved development of a global model across units or model updating by inclusion of spectra of the new population, using reference values estimated using the master unit.

\section{Introduction}

Spectrometers employing silicon photodiode array (PDA) detectors operating over the short wave NIR (780-1000 nm) are attractive for their low cost and relative ruggedness. However, units can vary in terms of wavelength accuracy and photometric response, impacting on the success of use of a model transfer between units. Any shift in the wavelength scale will create prediction errors when a model created on one unit is used with another unit, especially when there is high model weighting on spectral regions with high slopes (see, e.g., Fearn ${ }^{1}$ ). A wavelength dependant photometric response difference between units will result in spectra of different shapes, and thus also impact model transfer.

Calibration models can be developed for each device, but this is inefficient. Model transfer between instruments can be approached in three ways (Fearn, ${ }^{1}$ Andrew and Fearn, ${ }^{2}$ Soldado et al. $^{3}$ and lgne et al. ${ }^{4}$ ): (i) by making of a model that transfers without requiring standardisation, e.g. using spectral pre-treatments, selecting wavelengths ranges that exhibit spectral "stability", or by including data from multiple instruments in the calibration set; (ii) adjusting the model output such that it works on other instruments, e.g. simple slope

\section{Correspondence}

C. Hayes (c.hayes@cqu.edu.au)

doi: $10.1255 /$ nir2017.071

Citation: C. Hayes, K. Walsh and R. Lerud, "Calibration transfer between

short wave near infrared photodiode array instruments", in Proc. $18^{\text {th }}$ Int.

Conf. Near Infrared Spectrosc., Ed by S.B. Engelsen, K.M. Sørensen and F. van den Berg. IM Publications Open, Chichester, pp. 71-76 (2019). https://doi. org/10.1255/nir2017.071 and bias correction; (iii) correcting spectra from slave instruments to appear as they were acquired on master instrument, or (iv) transfer by orthogonal projection (TOP), with removal of spectral differences orthogonal to the calibration model.

The third approach in calibration transfer, of adjusting spectra from slave instruments to appear as master instrument spectra, has been addressed by a range of techniques. The spectral correction methods of spectral slope/bias correction (SSBC) and spectral bias correction, ${ }^{5}$ involve wavelength by wavelength simple linear regressions of spectra from each instrument. Other techniques, as reviewed by Fearn, ${ }^{1}$ include direct standardisation (DS), piecewise direct standardisation (PDS), double window piecewise direction standardisation (DWPDS), orthogonal signal correction (OSC), finite impulse response (FIR) and wavelet transform (WT).

Other approaches achieve an "implicit" orthogonalisation, such as the "repeatability" (REP) file concept. ${ }^{6}$ In this method, difference spectra (of the same sample scanned on multiple instruments) are scaled proportional to the ratio of the number of samples in the REP file and calibration set, then assigned an attribute value of zero and included in the calibration set.

\section{(C) 2019 The Authors}

This licence permits you to use, share, copy and redistribute the paper in any medium or any format provided that a full citation to the original paper is given.

ISBN: 978-1-906715-27-4 
In a similar approach, Saranwong and Kawano ${ }^{7}$ proposed a method for transfer of a partial least squares (PLS) calibration on apple total soluble solids (TSS) between two Foss NIRSystems 6500 spectrometers, in which the difference in the average spectra (second derivative absorbance) of a group of samples is used to adjust the slave instrument spectra by simple subtraction. This method effects a bias correction (difference spectrum multiplied by model coefficients) and cannot improve the SEP (bias corrected RMSEP) of the transferred model. In the case presented, the method was effective, with the resulting bias at the same level as the validation results on the master instrument.

There are few reports on calibration transfer work involving silicon PDA based spectrometers. Greensill et al. ${ }^{8,9}$ compared the performance of the seven techniques mentioned above and a model updating (MU) technique based on use of Kennard-Stone selected representative spectra. WT and MU proved to be the best methods, decreasing the RMSEP from 7.03 to $0.21 \%$ TSS, with very little difference in the RMSEP of the two techniques, although PDS and DWPDS methods also performed well. Hayes et al. ${ }^{10}$ investigated the impact of wavelength accuracy on PLS model performance for predicting apple TSS for SWNIR Si PDA based instruments and the benefit of improvement in wavelength calibration in concert with transfer routines for the porting of models between instruments. The instruments used employ a Zeiss MMS1 spectrometer with an interactance geometry. Wavelength assignment errors of up to $2.3 \mathrm{~nm}$ the range used in the PLS regressions on TSS were noted in some units. Both SEP and bias increased with inaccuracies in wavelength assignments. The transfer methodologies of PDS, TOP, $M U$ and the difference spectrum adjustment (DSA) of Saranwong and Kawano ${ }^{7}$ were trialled. The DSA method combined with new wavelength assignments and model updating gave results comparable to the performance of the master instrument and to models directly developed on the slave instruments $\left(r^{2}=0.95, \mathrm{SEP}_{-\mathrm{b}}=0.47\right.$ and bias $=-0.03 \%$ TSS, for a population of mean $=14.45$ and $\mathrm{SD}=1.64 \% \mathrm{w} / \mathrm{v})$. Although comparable, the DSA method combined with new wavelength assignments and model updating was preferred over PDS due to ease of implementation.

An issue of practical importance is that of the selection of the updating or transfer set. Bouveresse and Massart, ${ }^{11}$ Fearn $^{1}$ and de Noord ${ }^{12}$ suggest that the standardisation samples used should cover the same spectral intensity range as the model/prediction set, and those which cover a larger spectral range generally lead to poor results. Thus, the use of "real" or similar samples with the same spectral features as associated with samples to be predicted is advocated. However, the definition of "similar" requires consideration. For fruit, for example, does the PDS transfer set need to be based on the same fruit commodity or indeed the same fruit cultivar as that used in the model? Previous studies have used a subset of a calibration set for transfer.

Blanco et al. ${ }^{13}$ asserted that the wavelength accuracy is of more importance than absorbance response differences for model transfer across diode array UV-Vis spectrometers. The reverse is generally true for scanning monochromator NIR spectrometers. The wavelength to pixel assignments of PDA units are commonly calculated from a third order polynomial based on peak positions of mercury/argon lamp spectra. Peak assignment differences of $0.26 \mathrm{~nm}$ for a Si PDA with approximately $3.3 \mathrm{~nm}$ pixel dispersion have been reported. ${ }^{14}$ Given a wavelength resolution (full width half maximum) of around $10 \mathrm{~nm}$ in these instruments, and as SWNIR features are wide, a wavelength accuracy of around $1 \mathrm{~nm}$ would appear appropriate for matching of instruments. However, this is not so, e.g. Kaur et al. ${ }^{15}$ observed significant variation in Si PDA instrument performance (RMSEP) for (fruit dry matter content) models developed using the same wavelength range, a result ascribed to the variation in wavelength to pixel assignments of the two units.

The current study compares transfer methods for the porting of models between instruments, expanding an earlier consideration ${ }^{10}$ to include the methods of global modelling, global model updating and wavelength reassignment using non-biological samples. Our work is focussed on the application of SWNIRS to internal quality of fruit, so this study was framed by work with a spectrometer system and data set relevant to that application.

\section{Materials and methods}

Fruit spectra were acquired on six F-750 instruments (Felix Instruments, Camas, WA, USA) and destructively sampled for oven dry matter (\% DM). These instruments employ a Zeiss MMS1-NIR enhanced spectrometer in an interactance geometry. This geometry involves a probe receiving light to the MMS1 fibre optic placed in front of a halogen lamp mounted in a parabolic reflector, such 
that a shadow is cast on the sample, and the detected light is largely derived from this shadowed area. The MMS1 in five of the units employed a 58381 Si PDA detector (Hamamatsu Photonics K.K., Hamamatsu City, Shizuoka, Japan), while the older sixth unit employed a S4874 Si PDA detector (Hamamatsu Photonics K.K.). The change in array used in the MMS1 was made by Zeiss to reduce signal carryover between successive readouts and improve read-out time. The pixel to wavelength assignment in this unit differed to that of other units.

Two spectra were collected from two locations on each fruit. After spectra collection, $2 \mathrm{~cm}$ diameter by $1 \mathrm{~cm}$ deep cores of flesh were taken, skin removed and dry matter content (DM) assessed gravimetrically following drying at $65^{\circ} \mathrm{C}$ to constant weight.

The following fruit sets were scanned using all instruments. A population of mango cultivar Kensington Pride fruit ( $n=232, \mu=13.7, \sigma=1.6 \%$ DM) were used for calibration, with separate sets fruit of the same cultivar but different harvest dates used in validation ( $n=100$, $\mu=13.8, \sigma=1.5 \% \mathrm{DM})$, and all transfer methods ( $n=100$, $\mu=14.4, \sigma=1.3 \%$ DM). For further PDS transfer functions, spectra were collected of: (i) a set of cultivar Calypso fruit ( $n=280, \mu=16.6, \sigma=1.0 \%$ DM); (ii) R2E2 fruit ( $n=300, \mu=14.7, \sigma=1.4 \% \mathrm{DM}$ ) and (iii) apple fruit ( $n=176, \mu=12.9, \sigma=1.3 \% \mathrm{DM})$.

As HgAr lamp spectra could not be collected without disassembly of the instruments, the master instrument was arbitrarily defined as having "correct" pixel-to-wavelength assignments. Pixel-to-wavelength assignments for the $695-1014 \mathrm{~nm}$ range were generated using a fourth order polynomial fit to wavelength peaks of spectra of polypropylene, using wavelength assignments from the master unit.

PLS regression models were developed using MATLab R2014a (MathWorks Inc., Natick, MA, USA) with PLS toolbox 7.3 (Eigenvector Research Inc., Wenatchee, WA, USA), using mean centred 9-point Savitzky-Golay second derivative absorbance data, interpolated to $3 \mathrm{~nm}$ steps, and the wavelength range $732-936 \mathrm{~nm}$. The choice of number of PLS factors was based on minimisation of RMSECV, with seven factors chosen in most cases.

The PLS DM model developed for the master unit was used "direct" in prediction using unaltered spectra of other units, with results compared to those for several instrument standardisation techniques involving either spectral correction (PDS, DSA), global models or model updating (Table 1). For the DSA technique, the mean difference spectra was calculated using spectra of the Kensington Pride fruit transfer set, and used in adjustment of all validation set spectra. This was also combined with the new pixel-to-wavelength assignments, as described above. PDS was performed using the four different transfer sets listed above.

Several MU and global model approaches were trialled using the Kensington Pride transfer set: (i) a global model with all spectra of the calibration sets of units $A$ to $D$ (with E included only on its own); (ii) same as (i) with increased PLS factors (iii) a master unit based model, updated with spectra from the transfer sets of units A to D, (iv) same as (iii) with increased PLS factors, (v) model based on spectra of the master unit updated with spectra from the transfer set of the respective slave unit, (vi) a model based on spectra of the master unit, updated with spectra from the transfer set of the slave unit only, but predicted DM values from the master unit, (vii) slave unit based model, using spectra from the slave transfer set and predicted DM values from the master unit. The last two methods seek to improve practicality of implementation by avoiding the need for reference method analyses.

\section{Results and discussion}

Spectra of the same sample differed between units, with peak positions varying in wavelength assignment by up to $4 \mathrm{~nm}$ in the case of the older unit (unit E), as revealed in difference spectra (difference in the second derivative of absorbance spectra of a mango fruit between master and slave units, Figure 1). This result is similar to that

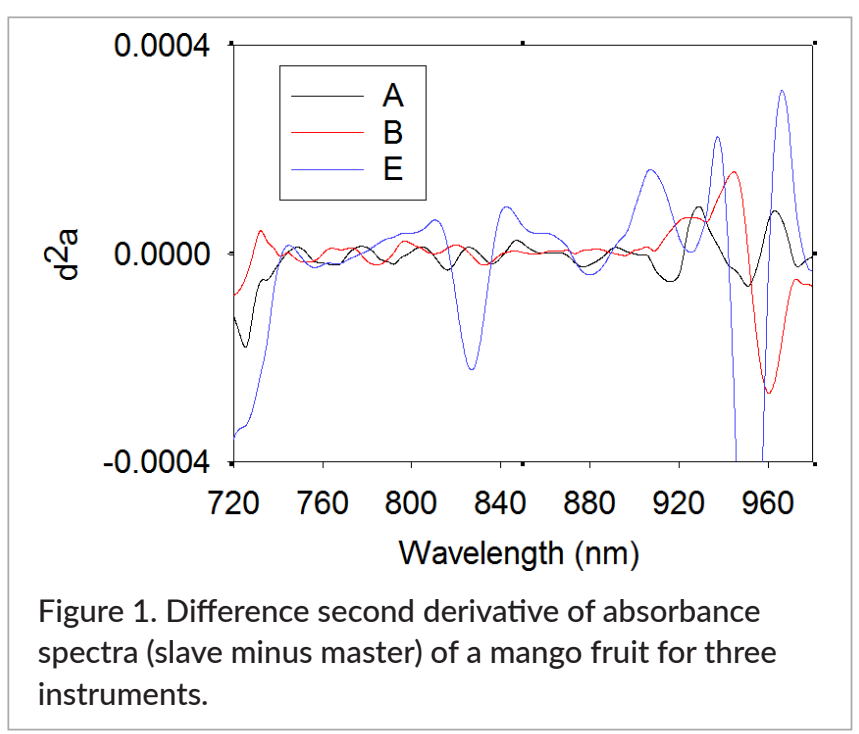


reported by Kaur et al. ${ }^{15}$ for the same spectrometer type. The variation in difference spectra between units $A$ to $E$ and the master unit is ascribed to the wavelength assignment issues, although detector wavelength sensitivity could also contribute to this observation. Due to this, when attempting methods (i)-(iv) above, spectra from unit $\mathrm{E}$ was only included when developing models for use on unit E.

The performance of models created on one unit and used in prediction of spectra collected on another unit

Table 1. (A) DM prediction statistics for models developed on a given unit using spectra of a calibration set of Kensington Pride fruit from respective units, used in prediction of an independent population of fruit; (B) DM prediction statistics for the calibration model transferred (using various methods) from the F-750 unit master to slave units A, B, C, D and E. For a given unit, best results are bolded ( $R^{2}$ values within 0.2 units, bias of 0.3 or less).

\begin{tabular}{|c|c|c|c|c|c|c|}
\hline & Master & $A$ & B & C & $\mathrm{D}$ & $\mathrm{E}$ \\
\hline & \multicolumn{6}{|c|}{$\mathrm{R}^{2}$} \\
\hline A. Calibration on single unit & 0.90 & 0.88 & 0.84 & 0.89 & 0.90 & 0.91 \\
\hline \multicolumn{7}{|l|}{ B. Transfer between units } \\
\hline Direct & 0.90 & 0.78 & 0.53 & 0.90 & 0.36 & 0.57 \\
\hline DSA & N/A & 0.78 & 0.53 & 0.90 & 0.36 & 0.57 \\
\hline DSA + wavelength reassignment & $N / A$ & 0.82 & 0.51 & 0.86 & 0.56 & 0.82 \\
\hline PDS & $N / A$ & 0.87 & 0.84 & 0.89 & 0.88 & 0.91 \\
\hline PDS(Calypso) & N/A & N/A & 0.82 & 0.88 & 0.85 & 0.89 \\
\hline PDS(R2E2) & $\mathrm{N} / \mathrm{A}$ & $\mathrm{N} / \mathrm{A}$ & 0.41 & 0.82 & 0.86 & 0.92 \\
\hline PDS (Apple) & N/A & 0.89 & 0.83 & 0.88 & 0.87 & 0.65 \\
\hline Global & 0.83 & 0.84 & 0.75 & 0.85 & 0.79 & 0.75 \\
\hline Global (10 PCs) & 0.90 & 0.89 & 0.85 & 0.92 & 0.86 & 0.79 \\
\hline Master model, global updating & 0.86 & 0.86 & 0.82 & 0.88 & 0.85 & 0.77 \\
\hline Master model, global updating (10 PCs) & 0.92 & 0.88 & 0.86 & 0.92 & 0.87 & 0.81 \\
\hline Individual model updating & 0.91 & 0.86 & 0.83 & 0.89 & 0.84 & 0.87 \\
\hline Individual model updating-master predicted DM & 0.90 & 0.85 & 0.82 & 0.87 & 0.83 & 0.86 \\
\hline \multirow[t]{2}{*}{ Individual models using master predicted DM } & 0.89 & 0.85 & 0.82 & 0.84 & 0.84 & 0.81 \\
\hline & \multicolumn{6}{|c|}{ Bias } \\
\hline A. Calibration on single unit & 0.01 & -0.14 & 0.16 & 0.12 & 0.05 & -0.02 \\
\hline \multicolumn{7}{|l|}{ B. Transfer between units } \\
\hline Direct & 0.01 & 1.94 & -0.26 & -2.81 & -1.68 & -17.64 \\
\hline DSA & $\mathrm{N} / \mathrm{A}$ & -0.37 & -0.57 & 0.22 & -0.63 & 0.03 \\
\hline DSA + wavelength reassignment & $\mathrm{N} / \mathrm{A}$ & 0.18 & -0.27 & 0.38 & -0.17 & 0.01 \\
\hline PDS & N/A & 0.01 & 0.09 & 0.14 & 0.20 & -0.01 \\
\hline PDS(Calypso) & N/A & $\mathrm{N} / \mathrm{A}$ & 0.04 & -0.07 & -0.09 & -0.66 \\
\hline PDS(R2E2) & N/A & $\mathrm{N} / \mathrm{A}$ & 0.40 & -0.87 & -0.03 & -0.30 \\
\hline PDS (Apple) & $N / A$ & -0.20 & 0.11 & 0.12 & 0.14 & -1.20 \\
\hline Global & -0.03 & 0.30 & 0.42 & -0.08 & -0.13 & 0.38 \\
\hline Global (10 PCs) & 0.02 & -0.04 & 0.19 & 0.07 & -0.16 & 0.37 \\
\hline Master model, global updating & 0.14 & 0.14 & 0.36 & -0.08 & -0.07 & 0.43 \\
\hline Master model, global updating (10 PCs) & -0.03 & -0.15 & 0.08 & 0.07 & -0.30 & 0.23 \\
\hline Individual model updating & -0.09 & -0.42 & -0.22 & -0.05 & -0.21 & 0.02 \\
\hline Individual model updating-master predicted DM & 0.01 & -0.22 & -0.07 & 0.10 & -0.07 & 0.12 \\
\hline Individual models using master predicted DM & -0.01 & 0.14 & 0.14 & 0.07 & -0.03 & -0.24 \\
\hline
\end{tabular}


was impacted both in terms of $r^{2}$ (and corresponding SEP-data not shown) and bias (Table 1, "Direct" results). The DSA method improved bias for all slave unit predictions, although bias magnitude $>0.5$ occurred in two cases, without affecting $\mathrm{R}^{2}$. In these two cases, the difference spectra, which was calculated using the transfer set, did not match the difference of the validation sets. The new pixel to wavelength assignment combined with DSA improved $R^{2}$ of unit $E$, as expected due to the discrepancy in wavelength calibration of this unit. All other transfer methods trialled were successful in decreasing bias (Table 1).

Prediction $\mathrm{R}^{2}$ was most consistently improved using PDS (based on a transfer set of the same variety of fruit as the calibration model), the Global (10 factors) and the Master model global updating (10 factors) approaches. However, the use of the same fruit variety for the PDS transfer set as used for the calibration set can be impractical due to seasonal availability of fruit. PDS using a related variety (Calypso) was successful across all units, however, use of fruit of the R2E2 variety for the transfer set proved unsuccessful on some units. Surprising, the use of apple spectra as the PDS training set proved quite successful, except on unit E. This latter result is believed to be due to the drastic difference in wavelength alignment of the units.

Except for unit E, a global model using spectra from all instruments gave satisfactory performance when the number of PLS factors was increased to 10, allowing the PLS calibration to model for instrument difference.

The performance of models based on individual unit model updating (model based on master calibration set plus transfer set from slave unit) was also comparable to that of PDS based models. Interestingly, use of DM values estimated by the master instrument (with no wet chemistry performed) for the transfer set (spectra collected on slave unit), gave acceptable performance. This approach has the advantage of being able to be implemented rapidly in field situations.

\section{Conclusion}

Best results ( $R^{2}$ and bias) were obtained using PDS using the same variety of fruit in calibration and transfer sets. This method is impractical due to seasonal availability of fruit. If the wavelength accuracy of the slave unit(s) is satisfactory, apples could be used to transfer the mango calibrations all year round (i.e. "out of mango season"). A global model across units or model updating, using reference values estimated using the master unit can be utilised in field situations.

\section{References}

1. T. Fearn, "Standardisation and calibration transfer for near infrared instruments: a review", J. Near Infrared Spectrosc. 9, 229 (2001). https://doi.org/10.1255/ jnirs.309

2. A. Andrew and T. Fearn, "Transfer by orthogonal projection: making near-infrared calibrations robust to between-instrument variation", Chemometr. Intell. Lab. Syst. 72, 51 (2004). https://doi.org/10.1016/j. chemolab.2004.02.004

3. A. Soldado, T. Fearn, A. Martínez-Fernández and B. de la Roza-Delgado, "The transfer of NIR calibrations for undried grass silage from the laboratory to on-site instruments: comparison of two approaches", Talanta 105, 8 (2013). https://doi.org/10.1016/j. talanta.2012.11.028

4. B. Igne, J.-M. Roger, S. Roussel, V. Bellon-Maurel and C.R. Hurburgh, "Improving the transfer of near infrared prediction models by orthogonal methods", Chemometr. Intell. Lab. Syst. 99, 57 (2009). https://doi. org/10.1016/j.chemolab.2009.07.007

5. Y. Roggo, L. Duponchel, B. Noe and J.P. Huvenne, "Sucrose content determination of sugar beets by near infrared reflectance spectroscopy. comparison of calibration methods and calibration transfer", J. Near Infrared Spectrosc. 10, 137 (2002). https://doi. org/10.1255/jnirs.330

6. M.O. Westerhaus, "Improving repeatability of NIR calibrations across instruments", in Third international Conference on Near Infrared Spectroscopy, Ed by R. Biston and N. Bartiaux-Thill. Brussels, Belgium, pp. 671 (1990).

7. S. Saranwong and S. Kawano, "A simple method of instrument standardisation for a near infrared sorting machine: the utilisation of average spectra as input vectors", J. Near Infrared Spectrosc. 12, 359 (2004). https://doi.org/10.1255/jnirs.444

8. C.V. Greensill and K.B. Walsh, "Calibration transfer between miniature photodiode array-based spectrometers in the near infrared assessment of manda- 
rin soluble solids content", J. Near Infrared Spectrosc. 10, 27 (2002). https://doi.org/10.1255/jnirs.318

9. C.V. Greensill, P.J. Wolfs, C.H. Spiegelman and K.B. Walsh, "Calibration transfer between PDA-based NIR spectrometers in the NIR assessment of melon soluble solids content", Appl. Spectrosc. 55, 647 (2001). https://doi. org/10.1366/0003702011952280

10. C. Hayes, K. Walsh and C. Greensill, "Improving calibration transfer between shortwave near infrared silicon photodiode array instruments", J. Near Infrared Spectrosc. 24, 59 (2016). https://doi. org/10.1255/jnirs.1194

11.E. Bouveresse, D.L. Massart and P. Dardenne, "Modified algorithm for standardization of nearinfrared spectrometric instruments", Anal. Chem. 67, 1381 (1995). https://doi.org/10.1021/ac00104a013
12. O.E. de Noord, "Multivariate calibration standardization", Chemometr. Intell. Lab. Syst. 25, 85 (1994). https://doi.org/10.1016/0169-7439(94)85037-2

13. M. Blanco, J. Coello, H. Iturriaga, S. Maspoch and E. Rovira, "Wavelength calibration transfer between diode array UV-visible spectrophotometers", Appl. Spectrosc. 49, 593 (1995). https://doi. org/10.1366/0003702953964084

14.Zeiss, Test Certificate. Carl Zeiss Jena GmbH, Jena (2002).

15. H. Kaur, R. Künnemeyer and A. McGlone, "Comparison of hand-held near infrared spectrophotometers for fruit dry matter assessment", J. Near Infrared Spectrosc. 25, 267 (2017). https://doi. org/10.1177/0967033517725530 